\title{
The origin of the most luminous Planetary Nebulae
}

\author{
Rebeca Galera-Rosillo ${ }^{1,2}$, Romano L. M. Corradi ${ }^{3}$, Bruce Balick ${ }^{4}$, \\ Karen Kwitter ${ }^{5}$, Antonio Mampaso ${ }^{1}$ and Jorge García-Rojas ${ }^{1}$ \\ ${ }^{1}$ Instituto de Astrofísica de Canarias, E-38206, La Laguna, Tenerife, Spain \\ email: rgr@iac.es \\ ${ }^{2}$ Departamento de Astrofísica, Universidad de La Laguna, E-38205, La Laguna, Tenerife, Spain \\ ${ }^{3}$ Gran Telescopio de Canarias, Centro de Astrofísica de la Palma, E-38712, S/C de Tenerife, \\ Spain \\ ${ }^{4}$ Astronomy Department, University of Washington, Seattle, WA, 98195-1580, USA \\ ${ }^{5}$ Department of Astronomy, Williams College, Williamstown, MA, 01267-2565, USA
}

\begin{abstract}
As part of a systematic effort to characterize the properties and progenitors of the most luminous planetary nebulae ( $\mathrm{PNe}$ ), we obtained a sample among the brightest $\mathrm{PNe}$ in two stellar systems of different metallicities: LMC $\left(\mathrm{Z} / \mathrm{Z}_{\odot} \sim 0.5\right)$ and $\mathrm{M} 31\left(\mathrm{Z} / \mathrm{Z}_{\odot} \sim 1\right)$ by means of a combined effort with the VLT and the 10mGTC. Modelling of these data will allow us to infer the masses of the stellar progenitors, gaining insights into the controversial origin of the universal cutoff of the Planetary Nebulae Luminosity Function (PNLF).
\end{abstract}

Keywords. Planetary Nebulae: general, galaxies: abundances, galaxies: M31,LMC, ISM: evolution

\section{Introduction}

The Planetary Nebulae Luminosity Function (PNLF), is the number density distribution of PNe in a system over a given absolute magnitude range (Jacoby 1980). It is an important standard candle on the extragalactic distance ladder, based on the empirical evidence that PN total L in the $\mathrm{O}[\mathrm{III}] 5007 \AA$ nebular emission line reaches a maximum value, $\mathrm{M}^{*}$, invariant with galaxy type and with only a small dependence on metallicity (Dopita et al. 1992, Ciardullo 2010). As PNe descend from progenitors present in any galactic subsystem, the PNLF method to determine extragalactic distances is equally effective for both spiral and elliptical galaxies, and has been applied to more than 50 galaxies.

Previous theoretical modelling suggested progenitor initial masses larger than $2 \mathrm{M} \odot$ (Schönberner et al. 2007, Méndez et al. 2008, Catalán et al. 2008) for these bright PNe, but relatively massive stars are too scarce or absent in old systems such as elliptical galaxies or haloes to produce the observed bright PNe. Alternative channels of PNe production that populate the bright end of the PNLF, as mergers, mass accretion in interacting binary stars or blue stragglers have been proposed (Ciardullo et al. 2005, Soker 2006). If they are able to provide the observed number of $\mathrm{M}^{*} \mathrm{PNe}$ is still controversial.

\section{Observations and inmediate objectives}

We present new very deep optical spectra of $8 \mathrm{PNe}$ within the bright tip of the $\mathrm{m}$ $5007 \AA$ PNLF in the M31 spiral galaxy, from OSIRIS at GTC, and 4 spectra of PNe at the Large Magellanic Cloud, within 0.6 mag from the PNLF cutoff from FORS2 at VLT. 
The first goal using these data is to obtain precise chemical abundances for a number of elements ( $\mathrm{He}, \mathrm{N}, \mathrm{O}, \mathrm{S}, \mathrm{Ne}, \mathrm{Ar}, \mathrm{Cl}$ ) to test the theoretical yield predictions for progenitors of different stellar masses, and determine - via Cloudy modelling of the spectra temperatures and luminosities for the central stars which can be located on evolutionary tracks of the H-R diagram yielding mass estimates.

In the case of the VLT data, we will try besides to tackle the abundance discrepancy problem: optical recombination lines (ORLs) provide abundance values that are systematically larger than those obtained using CELs. We will determine the abundance discrepancy factor (adf) in these $\mathrm{LMC} \mathrm{M}^{*} \mathrm{PNe}$, which will not only quantify the uncertainty in the real nebular abundances but will also provide an independent measure of the critical $\mathrm{C} / \mathrm{O}$ abundance ratio using ORLs.

\section{References}

Jacoby, G. H. 1980, ApJs,42, 1J

Dopita, M. A., Jacoby, G. H., \& Vassiliadis, E. 1992, Apj, 389, 27

Ciardullo, R. 2010 PASA, 27, 149

Schönberner, D., Jacob, R., Steffen, M., \& Sandin, C. 2007, A\&A A, 473, 467

Méndez, R. H., Teodorescu, A. M., Schönberner, D., Jacob, R., \& Steffen, M. 2008, Apj, 681, $325-332$

Catalán, S., Isern, J., García-Berro, E., \& Ribas, I. 2008, MNRAS, 387, 1693

Ciardullo, R., Sigurdsson, S., Feldmeier, J. J., \& Jacoby, G. H. 2005, Apj, 629, 499

Soker, N. 2006, Apj 640, 966 